\title{
Cops and Robber Game Without Recharging
}

\author{
Fedor V. Fomin* Petr A. Golovach ${ }^{\dagger} \quad$ Daniel Lokshtanov*
}

\begin{abstract}
Cops \& Robber is a classical pursuit-evasion game on undirected graphs, where the task is to identify the minimum number of cops sufficient to catch the robber. In this work, we consider a natural variant of this game, where every cop can make at most $f$ steps, and prove that for each $f \geq 2$, it is PSPACE-complete to decide whether $k$ cops can capture the robber.
\end{abstract}

\section{Introduction}

The study of pursuit-evasion games is driven by many real-world applications where a team of agents/robots must reach a moving target. The mathematical study of such games has a long history, tracing back to the work of Pierre Bouguer, who in 1732 studied the problem of a pirate ship pursuing a fleeing merchant vessel. In 1960s the study of pursuit-evasion games, moistly motivated by military applications like missile interception, gave a rise to the theory of Differential Games [10]. Besides the original military motivations, pursuitevasion games have found many applications reaching from law enforcement to video games and thus were studied within different disciplines and from different perspectives. The necessity of algorithms for pursuit tasks occur in many real-world domains. In the Artificial Intelligence literature many heuristic algorithms for variations of the problem like Moving Target Search have been studied extensively [7, 11, 12, 16, 17]. In computer games, for instance, computer-controlled agents often pursue human-controlled players and making a good strategy for pursuers is definitely a challenge [14]. The algorithmic study of pursuitevasion games is also an active area in Robotics [9, 21] and Graph Algorithms [15, 5].

One of the classical pursuit-evasion problems is the Man and Lion problem attributed to Rado by Littlewood in [13]: A lion (pursuer) and a man (evader) in a closed arena have equal maximum speeds. What tactics should the lion employ to be sure of his meal? See also for more recent results on this problem [3, 20]. The discrete version of the Man and Lion problem on graphs was introduced by Winkler and Nowakowski [18] and Quilliot [19]. Aigner and Fromme [1] initiated the study of the problem with several pursuers. This game, named Cops \& Robber, is played by two players: cop and robber on an undirected graph. The cop-player has a team of cops who attempt to capture the robber. At the beginning of the game cop-player selects vertices and put cops on these vertices. Then the robber player put the robber on a vertex. The players take turns starting with the cop-player. At every move each of the cops can be either moved to an adjacent vertex or kept on the same vertex. Similarly, the robber player responds by moving the robber to an adjacent vertex or keeping him on the same vertex. The cop-player wins if at some step of

\footnotetext{
${ }^{*}$ Department of Informatics, University of Bergen, N-5020 Bergen, Norway, emails: \{fedor.fomin|daniello\}@ii.uib.no.

${ }^{\dagger}$ School of Engineering and Computing Sciences, Durham University, South Road, DH1 3LE Durham, UK, email: petr.golovach@durham.ac.uk. Supported by EPSRC under project EP/G043434/1.
} 
the game he succeeds to catch the robber, i.e. to put one of his cops on a vertex occupied by the robber. The game was studied intensively and there is an extensive literature on this problem. We refer to surveys $[2,5]$ for references on different pursuit-evasion and search games on graphs.

In the game of Cops \& Robber there are no restrictions on the number of moves the players can make. Such model is not realistic for most of the applications: No lion can pursuit a man without taking a nap and no robot can move permanently without recharging batteries. In this work, we introduce more realistic scenario of Cops \& Robber, the model capturing the fact that each of the cops has a limited amount of power or fuel.

We also find the Cops \& Robber problem with restricted power interesting form combinatorial point of view because it generalizes the Minimum Dominating Set problem, one of the fundamental problems in Graph Theory and Graph Algorithms. Indeed, with fuel limit 1 every cop can make at most one move, then $k$ cops can win on a graph $G$ if and only if $G$ has a dominating set of size $k$. Thus two classical problems-Minimum Dominating Set (fuel limit is 1) and Cops \& Robber (unlimited fuel) are the extreme cases of our problem. It would be natural to guess that if the amount of fuel the cops posses is some fixed integer $f$, then problem is related to distance $f$ domination. Indeed, for some graph classes (e.g. for trees), the problems coincide. Surprisingly, the intuition that Cops \& Robber and Minimum f-Dominating Set (the classical NP-complete problem) should be similar from the computational complexity point of view is wrong. The main result of this paper is that the problem deciding if $k$ cops can win on an undirected graph is PSPACE-complete even for $f=2$. Another motivation for our work is the long time open question on the computational complexity of the Cops \& Robber problem (without power constrains) on undirected graphs. In 1995, Goldstein and Reingold [8], have shown that the classical Cops \& Robber game is EXPTIME-hard on directed graphs and conjectured that similar holds for undirected graphs. However, even NP-hardness of the problem was not known until very recently [4]. By our results, in the game on an $n$-vertex undirected graph if the number of steps each cop is allowed to make is at most some polynomial of $n$, then deciding if $k$ cops can win is PSPACE-complete. Another open question in the theory of pursuit-evasion games is on the possible length of winning strategies: Is it true that if $k$ cops can win on a graph then they also can do it by making a polynomial amount of steps? While we still do not know the answer to this question, but by our result the answer "yes" to this question would imply that the classical Cops \& Robber game is PSPACE-complete.

\section{Basic definitions and preliminaries}

We consider finite undirected graphs without loops or multiple edges. The vertex set of a graph $G$ is denoted by $V(G)$ and its edge set by $E(G)$, or simply by $V$ and $E$ if this does not create confusion. If $U \subseteq V(G)$ then the subgraph of $G$ induced by $U$ is denoted by $G[U]$. For a vertex $v$, the set of vertices which are adjacent to $v$ is called the (open) neighborhood of $v$ and denoted by $N_{G}(v)$. The closed neighborhood of $v$ is the set $N_{G}[v]=N_{G}(v) \cup\{v\}$. If $U \subseteq V(G)$ then $N_{G}[U]=\bigcup_{v \in U} N_{G}[v]$. The distance $\operatorname{dist}_{G}(u, v)$ between a pair of vertices $u$ and $v$ in a connected graph $G$ is the number of edges in a shortest $u, v$-path in $G$. For a positive integer $r, N_{G}^{r}[v]=\left\{u \in V(G): \operatorname{dist}_{G}(u, v) \leq r\right\}$. Whenever there is no ambiguity we omit the subscripts.

The Cops \& Robber game can be defined as follows. Let $G$ be a graph, and let $f>0$ be an integer. The game is played by two players: the cop-player $\mathcal{C}$ and the robber player $\mathcal{R}$, which make moves alternately. The cop-player $\mathcal{C}$ has a team of $k$ cops who attempt to 
capture the robber. At the beginning of the game this player selects vertices and put cops on these vertices. Then $\mathcal{R}$ put the robber on a vertex. The players take turns starting with $\mathcal{C}$. At every turn each of the cops can be either moved to an adjacent vertex or kept on the same vertex, and during the whole game each of the cops can be moved from a vertex to another vertex at most $f$ times in total. In other words, each of the cops has an amount of fuel which allows him to make at most $f$ steps. Let us note that several cops can occupy the same vertex at some move. Similarly, $\mathcal{R}$ responds by moving the robber to an adjacent vertex or keeping him on same vertex. It is said that a cop catches (or captures) the robber at some move if at that move they occupy the same vertex. Notice that even if a cop cannot move to adjacent vertex (run out of fuel), he is still active and the robber cannot move to the vertex occupied by the cop without being caught. The cop-player wins if one of his cops catches the robber. Player $\mathcal{R}$ wins if he can avoid such a situation, or equivalently, to survive for $k f+1$ moves, since it can be assumed that at least one cop is moved at each step (otherwise the robber can either keep his position or improve it). For an integer $f$ and a graph $G$, we denote by $c_{f}(G)$ the minimum number $k$ of cops sufficient for $\mathcal{C}$ to win on graph $G$.

We define the position of a cop as a pair $(v, s)$ where $v \in V(G)$ and $s$ is an integer, $0 \leq s \leq f$. Here $v$ is the vertex occupied by the cop, and $s$ is the number of moves along edges (amount of fuel) which the cop can do. The position of a team of $k$ cops (or position of cops $)$ is a multiset $\left(\left(v_{1}, s_{1}\right), \ldots,\left(v_{k}, s_{k}\right)\right)$, where $\left(v_{i}, s_{i}\right)$ is the position of the $i$-th cop. For the initial position, all $s_{i}=f$. The position of the robber is a vertex of the graph occupied by him.

We consider the following COPS AND RoBBER decision problem:

Input: A connected graph $G$ and two positive integers $k, f$.

Question: Is $c_{f}(G) \leq k$ ?

Let us finish the section on preliminaries with the proof of relations between Cops \& Robber and $r$-domination announced in Introduction. The Cops \& Robber problem with restricted power is closely related to domination problems. Let $r$ be a positive integer. A set of vertices $S \subset V(G)$ of a graph $G$ is called the $r$-dominating set if for any $v \in V(G)$, there is $u \in S$ such that $\operatorname{dist}(u, v) \leq r$. The $r$-domination number $\gamma_{r}(G)$ is the minimum $k$ such that there is an $r$-dominating set with at most $k$ vertices. Then $\gamma_{1}(G)$ is the domination number of $G$.

The proof of the following observation is straightforward.

Observation 1. For any connected graph $G, c_{1}(G)=\gamma_{1}(G)$.

For $f>1$, the values $c_{f}(G)$ and $\gamma_{f}(G)$ can differ arbitrary. Consider, for example, the graph $G$ which is the union of $k$ cycles of length $2 f+1$ with one common vertex. It can be easily seen that $\gamma_{f}(G)=1$ but $c_{f}(g)=k$. Still, for some graph classes (e.g. for trees) these numbers are equal. Recall, that the girth of a graph $G$, denoted by $g(G)$, is the length of a shortest cycle in $G$ (if $G$ is acyclic then $g(G)=\infty$ ).

Theorem 1. Let $f>0$ be an integer and let $G$ be a connected graph of girth at least $4 f-1$. Then $c_{f}(G)=\gamma_{f}(G)$.

Proof. The proof of $\gamma_{f}(G) \leq c_{f}(G)$ is trivial. To prove that $c_{f}(G) \leq \gamma_{f}(G)$, we give a winning strategy of $\gamma_{f}(G)$ cops. Suppose that $S$ is an $f$-dominating set in $G$ of size $\gamma_{f}(G)$. The cops are placed on the vertices of $S$. Suppose that the robber occupies a vertex $u$. Then the cops from vertices of $S \cap N_{G}^{2 f-1}$ move towards the vertex occupied by the robber 
at the current moment along the shortest paths. We claim that the robber is captured after at most $f$ moves of the cops. Notice that the robber can move at distance at most $f-1$ from $u$ before the cops make $f$ moves. Because $g(G) \geq 4 f-1$, the paths along which the cops move are unique. Suppose that the robber is not captured after $f-1$ moves of the cop-player, and the robber occupies a vertex $w$ after his $f-1$ moves. Since $S$ is an $f$-dominating set, there is a vertex $z \in S$ such that $\operatorname{dist}_{G}(w, z) \leq f$. Using the fact that $g(G) \geq 4 f-1$, and since the robber was not captured before, we observe that the cop from $z$ moved to $w$ along the shortest path between $z$ and $w$ and by his $f$-th move he has to enter $w$ and capture the robber.

\section{PSPACE-completeness}

It immediately follows from Observation 1 that it is NP-complete to decide whether $c_{1}(G) \leq k$. Here we prove that for any $f \geq 2$, the problem is much more difficult.

Theorem 2. For any $f \geq 2$, the COPS AND RoBBer problem is PSPACE-complete.

Proof. We reduce the PSPACE-complete Quantified Boolean Formula In ConjuncTIVe Normal Form (QBF) problem [6]. For a set of Boolean variables $x_{1}, x_{2}, \ldots, x_{n}$ and a Boolean formula $F=C_{1} \wedge C_{2} \wedge \cdots \wedge C_{m}$, where $C_{j}$ is a clause, the QBF problem asks whether the expression $\phi=Q_{1} x_{1} Q_{2} x_{2} \cdots Q_{n} x_{n} F$ is true, where for every $i, Q_{i}$ is either $\forall$ or $\exists$. For simplicity, we describe the reduction for the case $f=2$. For $f>2$, the proof uses the same ideas, but the construction is slightly more involved. We provide more details for the case $f>2$ right after we finish the proof of Lemma 5 .

Given a quantified Boolean formula $\phi$, we construct an instance $G, k$ of our problem in several steps. We first construct a graph $G^{(1)}$ and show that if the considered strategies are restricted to some specific conditions, then $\phi$ is true if and only if the cop-player can win on $G^{(1)}$ with a a specific number of cops.

Constructing $G^{(1)}$. For every $Q_{i} x_{i}$ we introduce a gadget graph $G_{i}$. For $Q_{i}=\forall$, we define the graph $G_{i}(\forall)$ with vertex set

$$
\left\{u_{i-1}, u_{i}, x_{i}, \bar{x}_{i}, y_{i}, \bar{y}_{i}, z_{i}\right\}
$$

and edge set

$$
\left\{u_{i-1} y_{i}, y_{i} u_{i}, u_{i-1} \bar{y}_{i}, \bar{y}_{i} u_{i}, z_{i} x_{i}, x_{i} y_{i}, z_{i} \overline{x_{i}}, \bar{x}_{i} y_{i}\right\} .
$$

For $Q_{i}=\exists$, we define $G_{i}(\exists)$ as the graph with vertex set

$$
\left\{u_{i-1}, u_{i}, x_{i}, \bar{x}_{i}, y_{i}, z_{i}\right\}
$$

and edge set

$$
\left\{u_{i-1} y_{i}, y_{i} u_{i}, z_{i} x_{i}, x_{i} y_{i}, z_{i} \overline{x_{i}}, \overline{x_{i}} y_{i}, x_{i} \bar{x}_{i}\right\} .
$$

The graphs $G_{i}(\forall)$ and $G_{i}(\exists)$ are shown in Fig 1 . Observe that the vertex $u_{i}$ appears both in the gadget graph $G_{i}$ and in the gadget $G_{i+1}$ for $i \in\{1,2, \ldots, n-1\}$. Let $U_{i}=\left\{u_{0}, \ldots, u_{i}\right\}$, $Y_{i}=\left\{y_{1}, \ldots, y_{i}\right\}$ and $\bar{Y}_{i}=\left\{\bar{y}_{j} \mid 1 \leq j \leq i\right\}$ for $1 \leq i \leq n$. The graph $G^{(1)}$ also has vertices $C_{1}, C_{2}, \ldots, C_{m}$ corresponding to clauses. The vertex $x_{i}$ is joined with $C_{j}$ by an edge if $C_{j}$ contains the literal $x_{i}$, and $\bar{x}_{i}$ is joined with $C_{j}$ if $C_{j}$ contains the literal $\bar{x}_{i}$. The vertex $u_{n}$ is connected with all vertices $C_{1}, C_{2}, \ldots, C_{m}$ by edges. An example of $G^{(1)}$ for $\phi=\exists x_{1} \forall x_{2}\left(x_{1} \vee \bar{x}_{2}\right) \wedge\left(\bar{x}_{1} \vee x_{2}\right)$ is shown in Fig 1.

We proceed to prove several properties of $G^{(1)}$. 


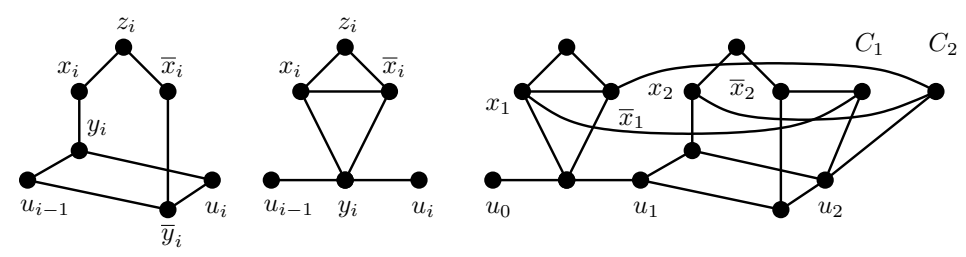

Figure 1: Graphs $G_{i}(\forall), G_{i}(\exists)$ and $G^{(1)}$

Lemma 1. Suppose that the robber can use only strategies with the following properties:

- he starts from $u_{0}$,

- he cannot remain in vertices $u_{0}, \ldots, u_{n}$,

- he moves along edges $u_{i-1} y_{i}, y_{i} u_{i}, u_{i-1} \bar{y}_{i}, \bar{y}_{i} u_{i}$ only in the direction induced by this ordering, i.e. these edges are "directed" for him.

Assume also that $n$ cops on $G^{(1)}$ use strategy with the following restrictions:

- they start from vertices $z_{1}, \ldots, z_{n}$,

- the cop on $z_{i}$ cannot move until the robber reaches vertices $y_{i}$ or $\bar{y}_{i}$.

Then $\phi=$ true if and only if $n$ cops have a winning strategy on $G^{(1)}$.

Proof. Assume that $\phi=$ true. We describe a winning strategy for the cop-player. The cops start by occupying vertices $z_{1}, \ldots, z_{n}$. Suppose that at some point during the game the robber moves to vertex $u_{i-1}$. Since he cannot stay in this vertex, we have that he has to move to $y_{i}$ or $\bar{y}_{i}$. If the robber moves to $y_{i}$ from $u_{i-1}$ of $G_{i}(\forall)$, then the cop occupying $z_{i}$ moves to $x_{i}$ and the corresponding variable $x_{i}$ is set to true. If the robber moves to $\bar{y}_{i}$, then the cop moves to $\bar{x}_{i}$ and we set $x_{i}=$ false. It means that for a quantified variable $\forall x_{i}$, the robber chooses the value of $x_{i}$. Notice that the robber cannot stay on $y_{i}$ or $\bar{y}_{i}$ because a cop which still has fuel occupies an adjacent vertex. Therefore he has to move to $u_{i}$. If the robber moves to $y_{i}$ of $G_{i}(\exists)$ from $u_{i-1}$, then the cop player replies by moving a cop from $z_{i}$ to $x_{i}$ or $\bar{x}_{i}$, and this represents the value of the variable $x_{i}$. Hence for a quantified variable $\exists x_{i}$, the cops choose the value of $x_{i}$. Then the robber is forced to move to $u_{i}$-it senseless for him to move to $x_{i}$ or $\bar{x}_{i}$ or stay in $y_{i}$. Since $\phi=$ true, we have that the cops in $G_{i}(\exists)$ gadgets can move in such a way that when the robber occupies the vertex $u_{n}$, every vertex $C_{j}$ has at least one neighbor occupied by a cop. If the robber moves to some vertex $C_{j}$ then a cop moves to $C_{j}$ and the robber is captured. Thus the cops win in this case.

Suppose that $\phi=$ false. We describe a winning strategy for the robber-player against cops occupying vertices $z_{1} \ldots, z_{n}$. The robber starts moving from $u_{0}$ toward the vertex $u_{n}$ along some path in $G^{(1)}$. Every time the robber steps on a vertex $y_{i}$ of $G_{i}(\forall)$, there should be a cop responding to this move by moving to $x_{i}$ from $s_{i}$. Otherwise the robber can stay in this vertex, and since cops from $z_{1}, \ldots, z_{i-1}$ do not have enough fuel to reach $y_{i}$ and the cops from $z_{i+1}, \ldots, z_{n}$ cannot move because of our restrictions, the robber-player wins in this case. By the same arguments, if the robber occupies $\bar{y}_{i}$, then the cop from $z_{i}$ has to move to $\bar{x}_{i}$. It means that in the same way as above the robber chooses the value of the variable $x_{i}$. Similarly, if the robber occupies the vertex $y_{i}$ in $G_{i}(\exists)$, then a cop is forced to move from $z_{i}$ to $x_{i}$ or $\bar{x}_{i}$, and this cop can choose which vertex from $x_{i}$ and $\bar{x}_{i}$ to occupy, 
and now the cop-player chooses the value of the variable $x_{i}$. Since $\phi=f a l s e$, we have that the robber can choose between $y_{i}$ and $\bar{y}_{i}$ in gadgets $G_{i}(\forall)$ such that no matter how the cop-player chooses to place the cops on $x_{i}$ or $\bar{x}_{i}$ in gadgets $G_{i}(\exists)$, when the robber arrives at $u_{n}$ at least one vertex $C_{j}$ is within distance two from vertices $x_{i}$ and $\bar{x}_{i}$ which were occupied by cops when the robbers visited $y_{i}$ or $\bar{y}_{i}$. Therefore, cops cannot reach this vertex. Then the robber moves to $C_{j}$ and remains there.

Now we are going to introduce gadgets that force players to follow the constraints on moves described in Lemma 1. Then we gradually eliminate all constraints. At first, we construct a gadget $F$ which forces a cop to occupy a given vertex and forbids him to leave it until some moment.

Constructing $F(W, t)$. Let $W$ be s set of vertices, and let $t \notin W$ be a vertex (we use these vertices to attach $F(W, t)$ to other parts of our constructions). We introduce four vertices $a, b, c, d$, join $a$ and $b$ with $t$ and the vertices of $W$ by edges, and join $c$ and $d$ with $t$ by paths of length two (see Fig 2).

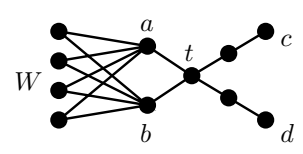

Figure 2: Graph $F(W, t)$

Properties of $F(W, t)$ are summarized in the following lemma.

Lemma 2. Let $H$ be a graph such that $V(H) \cap V(F(W, t))=W \cup\{t\}$. For any winning strategy for the cops on the graph $H^{\prime}=H \cup F(W, t)$, at least one cop have to be placed on vertices $V(F(W, t)) \backslash(W \cup\{a, b\})$ in the initial position, and if exactly one cop is placed there then he has to occupy $t$. Moreover, if one cop is placed on vertices $V(F(W, t)) \backslash(W \cup\{a, b\})$ and there are no other cops at distance two from $a, b$, then the cop cannot leave $t$ while the robber is on one of the vertices of $W$.

Proof. The first claim follows from the observation that at least one cop should be placed at distance at most two from $c$ and $d$. Otherwise the robber can occupy one of these vertices, and he cannot be captured. To prove the second claim, note that only the cop from $t$ can visit vertices $a$ and $b$. If the cop leaves $t$ then at least one of these vertices is not occupied by cops, and the robber can move there from vertices of $W$. After that he wins since no cop can reach this vertex.

Our next step is to force restrictions on strategies of the cop-player.

Constructing $G^{(2)}$. We consider the graph $G^{(1)}$. For each $1 \leq i \leq n$, the gadget $F\left(U_{i-1} \cup Y_{i-1} \cup \bar{Y}_{i-1}, z_{i}\right)$ is added. Denote the obtained graph by $G^{(2)}$ (see Fig 3).

Lemma 3. Suppose that the robber can use only strategies with the following properties:

- he starts from $u_{0}$ if cops are placed on $z_{1}, \ldots, z_{n}$,

- he cannot remain in vertices $u_{0}, \ldots, u_{n}$,

- he moves along edges $u_{i-1} y_{i}, y_{i} u_{i}, u_{i-1} \bar{y}_{i}, \bar{y}_{i} u_{i}$ only in the direction induced by this ordering, i.e. these edges are "directed" for him. 


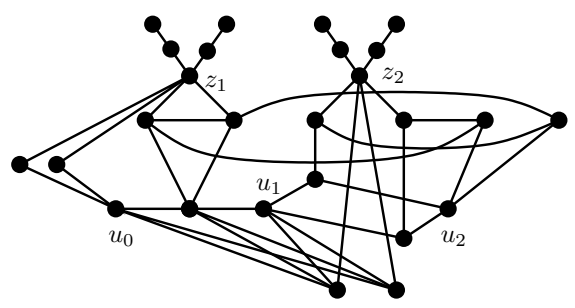

Figure 3: Graph $G^{(2)}$ for $\phi=\exists x_{1} \forall x_{2}\left(x_{1} \vee \bar{x}_{2}\right) \wedge\left(\bar{x}_{1} \vee x_{2}\right)$

Then $\phi=$ true if and only if $n$ cops have a winning strategy on $G^{(2)}$.

Proof. If $\phi=$ true then $n$ cops can use exactly the same winning strategy as in the proof of Lemma 3. We should only note that it makes no sense for the robber to move to vertices $a$ and $b$ of gadgets $F$ since he would be immediately captured. Suppose that $\phi=$ false. If the cops are not placed on $z_{1}, \ldots, z_{n}$, then by Lemma 2 the robber wins by staying in one of pendent vertices of gadgets $F$. If cops occupy vertices $z_{1}, \ldots, z_{n}$, then we can use the same winning strategy for the robber as the proof of Lemma 3. Indeed, by Lemma 2, the cop on $z_{i}$ cannot move until the robber reaches vertices $y_{i}$ or $\bar{y}_{i}$.

In the next stage we add a gadget that forces the robber to occupy $u_{0}$ in the beginning of the game.

Constructing $G^{(3)}$. We construct $G^{(2)}$. Then we add vertices $p, q, w_{1}, w_{2}$ and edges $w_{1}, w_{2}, w_{2} u_{0}$, and then join $p$ and $q$ with $w_{1}$ by paths of length two. Finally, we make $w_{1}$ to be adjacent to vertices $u_{1}, \ldots, u_{n}$ and $C_{1}, \ldots, C_{m}$. Denote the obtained graph by $G^{(3)}$ (see Fig 4).

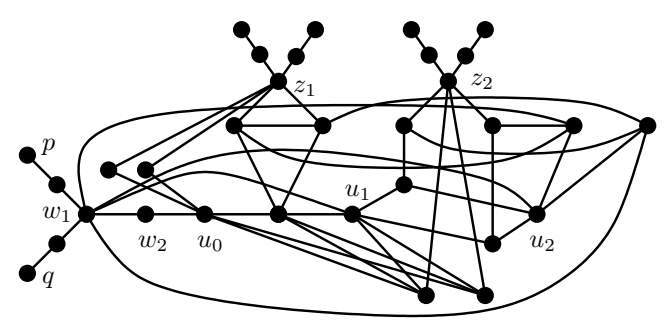

Figure 4: Graph $G^{(3)}$ for $\phi=\exists x_{1} \forall x_{2}\left(x_{1} \vee \bar{x}_{2}\right) \wedge\left(\bar{x}_{1} \vee x_{2}\right)$

Lemma 4. Suppose that the robber can use only strategies with the following properties:

- he cannot remain in vertices $u_{1}, \ldots, u_{n}$,

- he moves along edges $u_{i-1} y_{i}, y_{i} u_{i}, u_{i-1} \bar{y}_{i}, \bar{y}_{i} u_{i}$ only in the direction induced by this ordering, i.e. these edges are "directed" for him.

Then $\phi=$ true if and only if $n+1$ cops have a winning strategy on $G^{(3)}$.

Proof. Suppose that $\phi=$ true. We place $n+1$ cops on vertices $w_{1}, z_{1}, \ldots, z_{n}$. If the robber chooses vertices of $N_{G^{(3)}}\left[\left\{w_{1}, z_{1}, \ldots, z_{n}\right\}\right]$, then he can be right the next step. If he occupies vertices $p, q$ or pendent vertices of gadgets $F$, then he can be clearly captured in at most two steps. Suppose that the robber is placed on some vertex $y_{i}$ or $\bar{y}_{i}$. If he tries 
to move to $u_{i-1}$ or $u_{i}$, then he is captured by the cop from the vertex $w_{1}$. If he moves to some vertex $a$ or $b$ of gadget $F$ attached to a vertex $z_{j}, j>i$, then he is captured by the cop from $z_{j}$. Otherwise he is captured by the cop from the vertex $z_{i}$ in at most two steps. Thus the only remaining possibility for the robber to avoid the capture is to occupy $u_{0}$. In this case the robber from $w_{1}$ moves to $w_{2}$. Then the robber should leave the vertex $u_{0}$, and the cop-player can use the same strategy as before (see Lemma 3). Finally, the robber cannot move to $w_{1}$ from vertices $u_{1}, \ldots, u_{n}$ and $C_{1}, \ldots, C_{m}$, since he would be captured by the cop standing in $w_{2}$.

Suppose that $\phi=$ false. By Lemma 2 and by construction of $G^{(3)}$, we can assume that the cops are placed on $w_{1}, z_{1}, \ldots, z_{n}$ (otherwise the robber wins by choosing a pendent vertex within distance at least three from the cops). We describe a winning strategy for the robber-player against the cops occupying these vertices. The robber is placed on $u_{0}$. Then he waits until some cop is moved to an adjacent vertex. If the cop from $w_{1}$ is moved to some vertex different from $w_{2}$, then the robber responds by moving to $w_{2}$, and he wins by staying in this vertex. Suppose that a cop stays in the vertex $w_{1}$ and another cop, say the cop from $z_{i}$, moves to an adjacent vertex. The robber responds by moving to one of the vertices $a$ or $b$ of the gadget $F$ attached to $z_{i}$ and not occupied by cops. Then only the cop from $w_{1}$ can try to capture him by moving to some vertex $u_{j}, j<i$, but in this case the robber can return to $u_{0}$ and stay there. It remains to consider the case when a cop moves from $w_{1}$ to $w_{2}$, but now the robber can use the same winning strategy described before in Lemma 3.

Finally, we attach gadgets to force the remaining restrictions on actions of the robber.

Constructing $G^{(4)}$. We consider $G^{(3)}$. For each $1 \leq i \leq n$, we add vertices $f_{i}, g_{i}$ and the edge $f_{i} g_{i}$. Then $f_{i}$ is joined by edges with $u_{i}, y_{i}$ and $y_{i}$ (if it exists). Finally, we add the gadget $F\left(U_{i-1} \cup Y_{i} \cup \bar{Y}_{i}, g_{i}\right)$. The construction is shown in Fig 5 .

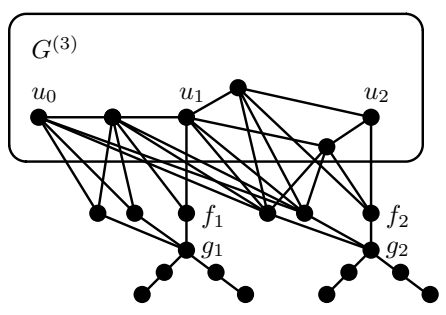

Figure 5: Graph $G^{(4)}$ for $\phi=\exists x_{1} \forall x_{2}\left(x_{1} \vee \bar{x}_{2}\right) \wedge\left(\bar{x}_{1} \vee x_{2}\right)$

Now we are in the position to prove the SPACE-hardness result.

Lemma 5. For the constructed graph $G^{(4)}$, we have $\phi=$ true if and only if $2 n+1$ cops have a winning strategy on $G^{(4)}$.

Proof. Suppose that $\phi=$ true. The cops are placed on the vertices $w_{1}, z_{1}, \ldots, z_{n}, g_{1}, \ldots, g_{n}$. The winning strategy for the cop-player is constructed as in Lemma 4 with one addition: if the robber reaches the vertex $u_{i}$, then a cop is moved from $g_{i}$ to $f_{i}$. Then the robber cannot stay in $u_{i}$ or move to $y_{i}$ or to $\bar{y}_{i}$. Notice also that if the robber is on $y_{i}$ or $\bar{y}_{i}$ then he cannot move to $u_{i-1}$ because he would be captured in one step by the cop from $f_{i-1}$ or $w_{2}$ if $i=1$.

Let $\phi=$ false. We can assume that the cops are occupying $w_{1}, z_{1}, \ldots, z_{n}, g_{1}, \ldots, g_{n}$ because otherwise the robber wins by selecting one of the pendent vertices at distance 2 
from one of the cop-free vertices. By Lemma 2, no cop can leave $g_{i}$ for $1 \leq i \leq n$, before the robber reaches the vertex $u_{i}$. But then the robber wins by making use exactly the same strategy we described in Lemma 4.

This concludes the proof of the PSPACE-hardness for $f=2$. For $f>2$, the proof is very similar and here we sketch only the most important differences. In particular, the graph $G^{(1)}$ should be modified in the following way: for each $1 \leq i \leq n$, we add a vertex $z_{i}^{\prime}$ and join it with the vertex $z_{i}$ by a path of length $f-2$. For this graph, it is possible to prove the claim similar to Lemma 1 with the difference that the cops should start from vertices $z_{1}^{\prime} \ldots, z_{n}^{\prime}$ and with additional condition that the robber cannot leave $u_{0}$ until some cop enters one of the vertices $z_{0}, \ldots, z_{n}$, and then to "enforce" special strategies for the players.

To complete the proof of the theorem, it remains to show that our problem is in PSPACE.

Lemma 6. For every integers $f, k \geq 1$ and an n-vertex graph $G$, it is possible to decide whether $c_{f}(G) \leq k$ by making use of space $O\left(k f n^{O(1)}\right)$.

Proof. The proof is constructive. We describe a recursive algorithm which solves the problem. Note that we can consider only strategies of the cop-player such that at least one cop is moved to an adjacent vertex. Otherwise, if all cops are staying in old positions, the robber can only improve his position.

Our algorithm uses a recursive procedure $W(P, u, l)$, which for a non negative integer $l$, position of the cops $P=\left(\left(v_{1}, s_{1}\right), \ldots,\left(v_{k}, s_{k}\right)\right)$ such that $l=s_{1}+\ldots+s_{k}$, and a vertex $u \in V(G)$, returns true if $k$ cops can win starting from the position $P$ against the robber which starts from the vertex $u$, and the procedure returns false otherwise. Clearly, $k$ cops can capture the robber on $G$ if and only if there is an initial position $P_{0}$ such that for any $u \in V(G), W\left(P_{0}, u, l\right)=$ true for $l=k f$.

If $l=0$ then $W(P, u, l)=$ true if and only if $u=v_{i}$ for some $1 \leq i \leq k$. Suppose that $l>0$. Then $W(P, u, l)=$ true in the following cases:

- $u=v_{i}$ for some $1 \leq i \leq k$,

- $u \in N_{G}\left(v_{i}\right)$ and $s_{i}>0$ for some $1 \leq i \leq k$,

- there is a position $P^{\prime}=\left(\left(v_{1}^{\prime}, s_{1}^{\prime}\right), \ldots,\left(v_{k}^{\prime}, s_{k}^{\prime}\right)\right)$ such that the cops can go from $P$ to $P^{\prime}$ in one step, and for any $u^{\prime} \in N_{G}[u], W\left(P^{\prime}, u^{\prime}, l^{\prime}\right)=$ true where $l^{\prime}=s_{1}^{\prime}+\ldots+s_{k}^{\prime}<l$.

Since all positions can be listed (without storing them) by using polynomial space, the number of possible moves of the robber is at most $n$, and the depth of the recursion is at most $k f$, the algorithm uses space $O\left(k f n^{O(1)}\right)$.

Now the proof of the theorem follows from Lemmata 5 and 6 .

Notice that the PSPACE-hardness proof is also holds for the case when $f$ is a part of the input. However, our proof only shows that the problem is in PSPACE only for $f=n^{O(1)}$. 


\section{Conclusion}

In this paper we introduced the variant of the Cops \& Robber game with restricted resources and have shown that the problem is PSPACE-complete for every $f>1$. In fact, our proof also shows that the problem is PSPACE-complete even when $f$ is at most some polynomial of $|V(G)|$. One of the long standing open questions in Cops \& Robber games, is the computational complexity of the classical variant of the game on undirected graphs without restrictions on the power of cops. In 1995, Goldstein and Reingold [8] conjectured that this problem is EXPTIME-hard. On the other hand, we do not know any example, where to win cops are required to make exponential number of steps (or fuel). This lead to a very natural question: Is it true that cops never need exponential amount of fuel? By our result, if the answer to this purely combinatorial question is "Yes", then the classical Cops \& Robber game without fuel restrictions on undirected graphs is PSPACE-complete.

\section{References}

[1] M. Aigner And M. Fromme, A game of cops and robbers, Discrete Appl. Math., 8 (1984), pp. $1-11$.

[2] B. Alspach, Searching and sweeping graphs: a brief survey, Matematiche (Catania), 59 (2006), pp. 5-37.

[3] A. Dumitrescu, I. Suzuki, And P. Zylinski, Offline variants of the "lion and man" problem, in Proceedings of the 23d annual symposium on Computational Geometry (SCG 07), New York, NY, USA, 2007, ACM, pp. 102-111.

[4] F. V. Fomin, P. A. Golovach, and J. Kratochvíl, On tractability of cops and robbers game, in IFIP TCS, G. Ausiello, J. Karhumäki, G. Mauri, and C.-H. L. Ong, eds., vol. 273 of IFIP, Springer, 2008, pp. 171-185.

[5] F. V. Fomin And D. M. Thilikos, An annotated bibliography on guaranteed graph searching, Theor. Comput. Sci., 399 (2008), pp. 236-245.

[6] M. R. Garey and D. S. Johnson, Computers and intractability, W. H. Freeman and Co., San Francisco, Calif., 1979. A guide to the theory of NP-completeness, A Series of Books in the Mathematical Sciences.

[7] M. Goldenberg, A. Kovarsky, X. Wu, and J. Schaeffer, Multiple agents moving target search, in Proceedings of the 18th international joint conference on Artificial Intelligence (IJCAI 03), San Francisco, CA, USA, 2003, Morgan Kaufmann Publishers Inc., pp. 1536-1538.

[8] A. S. Goldstein And E. M. Reingold, The complexity of pursuit on a graph, Theoret. Comput. Sci., 143 (1995), pp. 93-112.

[9] L. J. Guibas, J. Claude Latombe, S. M. Lavalle, D. Lin, and R. Motwani, A visibility-based pursuit-evasion problem, International Journal of Computational Geometry and Applications, 9 (1996), pp. 471-494.

[10] R. IsaAcs, Differential games. A mathematical theory with applications to warfare and pursuit, control and optimization, John Wiley \& Sons Inc., New York, 1965.

[11] T. Ishida And R. E. Korf, Moving target search, in Proceedings of the International joint conference on Artificial Intelligence (IJCAI 91), 1991, pp. 204-211.

[12] T. IshidA AND R. E. KorF, Moving-target search: A real-time search for changing goals, IEEE Trans. Pattern Anal. Mach. Intell., 17 (1995), pp. 609-619.

[13] J. E. Littlewood, Littlewood's miscellany, Cambridge University Press, Cambridge, 1986. Edited and with a foreword by Béla Bollobás. 
[14] P. K. K. Loh and E. C. Prakash, Novel moving target search algorithms for computer gaming, Comput. Entertain., 7 (2009), pp. 1-16.

[15] N. Megiddo, S. L. Hakimi, M. R. Garey, D. S. Johnson, and C. H. Papadimitriou, The complexity of searching a graph, J. Assoc. Comput. Mach., 35 (1988), pp. 18-44.

[16] C. Moldenhauer and N. R. Sturtevant, Evaluating strategies for running from the cops, in Proceedings of the 21st International Joint Conference on Artificial Intelligence (IJCAI 2009), 2009, pp. 584-589.

[17] _ Optimal solutions for moving target search, in Proceedings of the 8th International Joint Conference on Autonomous Agents and Multiagent Systems (AAMAS 2009), IFAAMAS, 2009, pp. 1249-1250.

[18] R. Nowakowski and P. Winkler, Vertex-to-vertex pursuit in a graph, Discrete Math., 43 (1983), pp. 235-239.

[19] A. Quilliot, Some results about pursuit games on metric spaces obtained through graph theory techniques, European J. Combin., 7 (1986), pp. 55-66.

[20] J. SGall, Solution of David Gale's lion and man problem, Theor. Comput. Sci., 259 (2001), pp. 663-670.

[21] K. Sugihara, I. Suzuki, And M. Yamashita, The searchlight scheduling problem, SIAM J. Comput., 19 (1990), pp. 1024-1040. 